Tropical Journal of Pharmaceutical Research January 2021; 20 (1): 1-9

ISSN: $1596-5996$ (print); 1596-9827 (electronic)

(C) Pharmacotherapy Group, Faculty of Pharmacy, University of Benin, Benin City, 300001 Nigeria.

Available online at http://www.tjpr.org

Original Research Article

http://dx.doi.org/10.4314/tjpr.v20i1.1

\title{
Long non-coding RNA KCNQ1 opposite transcript 1 (KCNQ1OT1) regulates cell proliferation and invasion via targeting miR-124-3p in gastric carcinoma
}

\author{
Xinying Ye ${ }^{1}$, Hongfu Liu ${ }^{1}$, Zhen Zeng ${ }^{2 *}$ \\ ${ }^{1}$ Department of General Surgery, The First Affiliated Hospital of Gannan Medical University, PR China, ${ }^{2} C T$ Room, The First \\ Affiliated Hospital of Gannan Medical University, PR China
}

*For correspondence: Email: er56623@126.com; Tel: +86-18726520999

Sent for review: 13 April 2020

Revised accepted: 20 September 2020

\begin{abstract}
Purpose: Long non-coding RNA (IncRNA) KCNQ1OT1 (KCNQ1 opposite strand/antisense transcript 1) has been associated with many kinds of cancers. However, its role hasn't been researched much in gastric cancer. The present study investigated the role and mechanism of KCNQ1OT1 in gastric cancer. Methods: Quantitative-PCR assays was used to measure KCNQ1OT1 and miR-124-3p expression in gastric cancer tissues and cells. Transfection assays were conducted to evealuate the regulation of KCNQ1OT1 or miR-124-3p expression in vitro. CCK-8 assays examined cell proliferation and Transwell invasion assays checked cell invasion abilities due to the changes of the key genes. On the other hand, luciferase reporter assays assisted in verifying the binding between KCNQ1OT1 and miR-124-3p. To explore the mechanism of KCNQ1OT1 in gastric cancer, we checked the independent influence of .KCNQ1OT1 or miR-124-3p on cellular functions and also examined the interplay between the two genes in gastric cancer cell lines.

Results: The findings showed that KCNQ1OT1 had high expression while miR-124-3p had low expression in gastric cancer tissues and cells in comparison with normal ones. KCNQ1OT1 was found to bind and target miR-124-3p. KCNQ1OT1 was shown to promote cell proliferation and invasion while miR-124-3p inhibit. Furthermore, the inhibition of miR-124-3p could partially reverse cell function changes induced by the knockdown of KCNQ1OT1 including cell viability, cell invasion and EMT process.

Conclusion: KCNQ1OT1 was a target of miR-124-3p and reduction in its expression led to an increase of miR-124-3p and inhibited the proliferation, invasion and EMT in Gastric Cancer. These results provided evidence that KCNQ1OT1 promoted the growth and invasion of the cells via targeting miR124-3p in GC.
\end{abstract}

Keywords: KCNQ1OT1, miR-124-3p, gastric carcinoma, proliferation, invasion

This is an Open Access article that uses a fund-ing model which does not charge readers or their institutions for access and distributed under the terms of the Creative Commons Attribution License (http://creativecommons.org/licenses/by/4.0) and the Budapest Open Access Initiative (http://www.budapestopenaccessinitiative.org/read), which permit unrestricted use, distribution, and reproduction in any medium, provided the original work is properly credited.

Tropical Journal of Pharmaceutical Research is indexed by Science Citation Index (SciSearch), Scopus, International Pharmaceutical Abstract, Chemical Abstracts, Embase, Index Copernicus, EBSCO, African Index Medicus, JournalSeek, Journal Citation Reports/Science Edition, Directory of Open Access Journals (DOAJ), African Journal Online, Bioline International, Open-J-Gate and Pharmacy Abstracts

(C) 2021 The authors. This work is licensed under the Creative Commons Attribution 4.0 International License 


\section{INTRODUCTION}

Gastric cancer has remained to be a huge menace and a concern for public health in the world with a high number of deaths resulting from it. It ranks fourth among the world's malignancies and has been reported as the second cause of deaths resulting from malignancies in the world $[1,2]$. Pathogenesis of cancer has been found to be complex and some study suggests that cancer development and growth are influenced by association of genes and the environment and the results from environmental effects and the susceptibility to develop tumors. Presently, the gold standard therapy which can cure gastric cancer is radical surgical resection with standardized D2-lymphadenectomy. The invasive ability of the virus has necessitated that new non-invasive methods should be sought after so as to enable the disease to be detected early.

Long non-coding RNA (IncRNA) are a distinct class of mRNA look alike transcripts that are longer than $\sim 200 \mathrm{nt}$ to $\sim 100 \mathrm{~kb}$ long [3]. They do not possess the ability to protein code and are thought to take part in many biological processes $[4,5]$. Many evidences have pointed to the expression of IncRNA in many cancers and thus many scientific studies have been conducted to find out the role functional IncRNA in the human malignant tissues

LncRNA also have been determined to have the ability to check the gene expression using some mechanisms like transcription, post-transcription, genomic imprinting, chromatin modification and protein function regulation.

Deregulated IncRNA has been shown to have a hand in the development of several different types of cancers in human beings such as breast cancer, colon cancer, bladder cancer and other types [6]. Malat1 for example can work, as an oncogene, to promote metastasis and growth of lung cancer and this can therefore be used as a bio marker in deciding the patients' prognosis [7]. Long non coding RNAs have also been linked to invasion and metastasis in cancers of the head and neck [8]. However, to date the functions and behavior of IncRNA in tumor growth is yet to be clearly understood. It is therefore vital to find the novel IncRNA as biomarkers for intervention therapeutically.

The relationship of KCNQ1OT1 and miR-124-3p is known but there are currently no publications on their role and expression in gastric cancer. In this study, we determined the amount of KCNQ10T1 and miR-124-3p in gastric cancer tissues and the different cell lines. We then analyzed the cell proliferation and invasion of KCNQ10T1. This enabled us to analyze the association between KCNQ10T1 and miR-124$3 p$ and their clinicopathological significance in gastric cancer.

\section{EXPERIMENTAL}

\section{Patients and tissue samples}

We gathered and collected 55 GC and 55 normal gastric tissue specimens over a period of five years from patients who agreed to undergo surgery in specific times at Second Hospital of Lanzhou University, China. These situations were all determined to be GC by the tests carried out in the hospital and to our knowledge they were not taken through any type of radiotherapy and/or chemotherapy before taking part in the study. Liquid nitrogen was used to freeze the specimens as required and then they were then kept at temperatures as low as $-80^{\circ} \mathrm{C}$. This research was approved by the Medical Ethics Committee of the Second Hospital of Lanzhou University, China and each participant signed a medically informed consent before we proceeded.

\section{Cell lines and cell culture}

The AGS, BGC, Hep3B, LMSU and MKN45, human $\mathrm{GC}$ cell lines which were bought from the American Type Culture Collection were all maintained in our laboratories. LO2 human normal cell also was bought from the Cell Bank of Type Culture Collection in Shanghai. All the cells used in the research were cultured in Iscove's modified Dulbecco's medium (IMDM; Gibco, Beijing, China). All the cells were kept in a humidified incubator at $37 \circ \mathrm{C}$ having $5 \% \mathrm{CO} 2$.

\section{Cell Transfection}

GenePharma Co., Ltd helped with construction of three sets of KCNQ10T1 siRNA which were shortly seeded in a 6 -well plate. Once the cell amount was at $70-80 \%$ confluency, the cells were taken and then transfected with KCNQ1OT1siRNA1, siRNA2, siRNA3 with the help of riboFECTTM CP transfection reagents following the instructions from the manufacturer.We used qRT-PCR to measure the expression of KCNQ1OT1R1L4 in the cells. SinceKCNQ10T1siRNA1 showed arelatively higher effective ability to knock down the expression of KCNQ10T1we chose to use this vector for the rest of the experiments. We also made shRNA sequence that focused onKCNQ1OT1 which were then ligated into 
pENTR vector (sh KCNQ1OT1) after being annealed.

\section{Quantitative real-time PCR}

We used TRIzol reagent (Takara Bio, Inc., Shiga, Japan), to extract and isolate all the required cellular RNA from the cancer cells and tissues used. For KCNQ1OT1, RNA we used the Prime Script RT-PCR Kit ( TaKaRa, Dalian, China) to transcribe it into CDNA in accordance with instructions from the manufacturer. For miR-124$3 p$ on the other hand, RNA was reversely transcribed with the help of M-MLV Reverse Transcriptase (Invitrogen, Carlsbad, CA, USA). The amounts of KCNQ1OT1 and miR-124-3p were then measured using qRT-PCR with a SYBR GREEN MIX kit all following the manufacturer's protocols. Primers sequences of KCNQ1OT1: forward, 5'- GCACTCTGGGTCC TGTTCTC-3'; reverse, 5'-CACTTCCCTGCCTC CTACAC-3'[9]. Primers sequences of miR-106a5p: forward, 5'-GATGCTCAAAAAGTGCTTACA GTGCA -3' and reverse, 5'-TATGGTTGTTCT GCTCTCTGTCTC -3'[10]. Primers sequences of GAPDH: forward, 5'-AAGGTGAAGGTCGGA GTCAA-3'; reverse, 5'-AATGAAGGGGTCA TTGATGG-3'. The experiments were all done in triplicate. $2-\Delta \Delta$ Ct method was used to quantify the gene expression.

\section{Cell viability assay}

AGS and BGC transfection with siNC or siKCNQ1OT1 were seeded into 96-well plates at a density of $4 \times 103$. After cultured in DMEM with $10 \%$ FBS for a night, the Cell Counting Kit-8 (Beyotime, Shanghai, China) was used to check cell viability after transfection based on the product manual. The results were checked at 24 , 48,72 and $96 \mathrm{~h}$. The absorbance was tested at $450 \mathrm{~nm}$ wave length and the cell viability curve was made according to the values recorded.

\section{Western blot assay}

After the GC cell lines AGS and BGC-823 were centrifuged at $2500^{*} \mathrm{~g}$ for five minutes, the cells were washed twice in cold PBS and then kept in RIPA Lysis and Extraction Buffer (Thermo Scientific, Massachusetts, USA) environment. The mixture was shaken for 15 minutes on ice and then centrifuged at $14000^{*} \mathrm{~g}$ for fifteen minutes so as to separate cell pieces. The supernatant was electrophoresis in 10\% SDSPAGE gel (Beyotime, Shanghai, China). Then the extracted proteins were transferred to 0.45 um PVDF membranes (Millipore, Billerica, MA, USA) and was blocked with $5 \%$ skim milk for a night around $4 \circ \mathrm{C}$. E-Cadherin Mouse Monoclonal
Antibody( 1:800), N-Cadherin Rabbit Polyclonal Antibody (1:800), Vimentin Mouse Monoclonal Antibody (1:800) and GAPDH Mouse Monoclonal Antibody( 1:800) were used as primary antibodies (Beyotime, Shanghai, China). Rabbit Anti-pan Cadherin antibody (1:200, Abcam, Cambridge, UK), Mouse Anti-pan Cadherin antibody (1:1000, Abcam, Cambridge, UK) and Goat Anti-Mouse IgG (1:1000, Beyotime, Shanghai, China) were used as secondary antibodies. The membranes were finally put forward to BeyoECL Plus Kit (Beyotime, Shanghai, China) adopted to reach the grey bands. The Western blot assay in this research was to examine $\mathrm{E}$-cadherin, $\mathrm{N}$-cadherin and vimentin with normalization to housekeeping gene GAPDH.

\section{Dual Luciferase assay}

The GC cell lines AGS and BGC-823AGScells were seeded and cultured in a 96-well plate. The pGL6 plasmid (Beyotime, Shanghai, China)was used to build cell models. PGL6 emptey vector, PGL6- KCNQ1OT1mut and PGL6- KCNQ1OT1wt were formed and transfected to AGS and BGC-823AGS cell lines for different groups. Then the cells were cotransfected with miR-124$3 p$ mimics and miR-124-3p mimics control . Luciferase activity was observed two days later than co-transfection. The Luciferase Assay system (Promega, Beijing, China) was adopted to measure the luciferase activity of different groups. Experiments were done independently three times.

\section{Transwell invasion assay}

The transwell invasion assay examined cell invasive ability of GC cells. Briefly, we used the Matrigel-coated chambers (BD Biosciences, San José, CA) with 8- $\mu \mathrm{m}$ pores to analyze the invasion ease of the GC cells. The cells that had been transfected $(2 \times 105)$ were planted in the upper invasion chamber containing medium deprived of serum. The lower invasion chamber contained RPMI 1640 supplemented with 10\% FBS to activate cell invasion thus the cells that moved to the lower invasion chamber after a $48 \mathrm{~h}$-incubation at $37^{\circ} \mathrm{C}$, period were joined together with $4 \%$ paraformaldehyde, with $0.1 \%$ crystal violet used for staining all for 30 minutes. Olympus light microscope (Tokyo, Japan) were used and quantities of cells were calculated.

\section{Statistical analysis}

The experiments in this study were done in triplicate and all data expressed as mean and $\mathrm{SD}$. The data was done via using the software 
Statistical Program for Social Sciences (SPSS) 19.0. Graphpad Prism 8.20 was used to process statistical data derived from the assays.

\section{RESULTS}

\section{Expression of KCNQ1OT1and miR-124-3p in gastric cancer}

First, qRT-PCR assays confirmed high expression of KCNQ1OT1 and low miR-124-3pin 55 pairs of tissues that were collected from gastric cancer patients after surgery. Signed consents were given by the respective patients and under supervision of Second Hospital of Lanzhou University. The results as presented in (Figure 1A) shows that KCNQ1OT1was relatively upregulated in gastric tissue specimens and was downregulated in the normal cells. On the contrary, miR-124-3p was lowly expressed in the cancer tissues (Figure 1B). To further find out the expression of KCNQ1OT1 and miR-124-3p in gastric cancer, we detected their levels using qRT-PCR in gastric cancer cell lines LMSU, MKN45, AGS and BGC-823 and the normal cell line LO2. BGC-823 had the highest expression of KCNQ1OT1 and the lowest expression of miR124-3p as compared to the other cell lines and

A

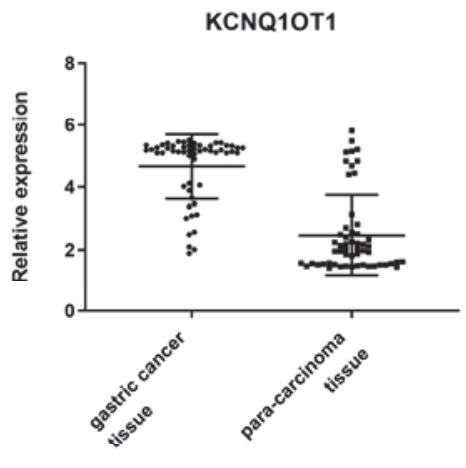

C

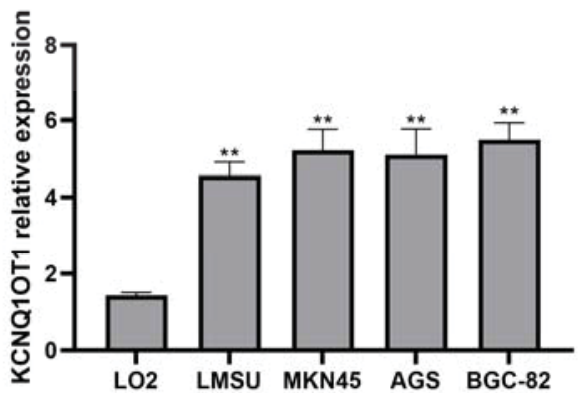

the normal cell line LO2 (Figure 1C-D). These findings may suggest that high expression of KCNQ10T1 or low miR- 124-3pmight promote gastric cancer and there was interplay between KCNQ1OT1 and miR-124-3p in gastric cancer.

\section{Downregulation of KCNQ1OT1 inhibited cell proliferation and cell viability in GC cells}

After we determined that KCNQ10T1 was upregulated in the gastric cancer cells lines, we performed a number of experiments to study the biological role of KCNQ10T1 in GC cell lines. To do this we first transfected AGS and BGC cells with KCNQ1OT siRNA1,KCNQ1OT1 siRNA2 and KCNQ1OT siRNA3and RT-qPCR checked the knockdown efficiency. KCNQ1OT1siRNA1 showed a more effective knockdown ability of KCNQ1OT1 in AGS and BGC cells (Figure 2A). Hence, we used KCNQ1OT1siRNA1 to knock down KCNQ1OT1 expression in AGS and BGC cells for following assays. To determine effect that KCNQ1OT1 played on GC cells, we did CCK8 assay first and compared it with NC-siRNA and found that si-KCNQ1OT1 inhibited cell viability compared to the control group (Figure 2B and $2 \mathrm{C}$ ).

B

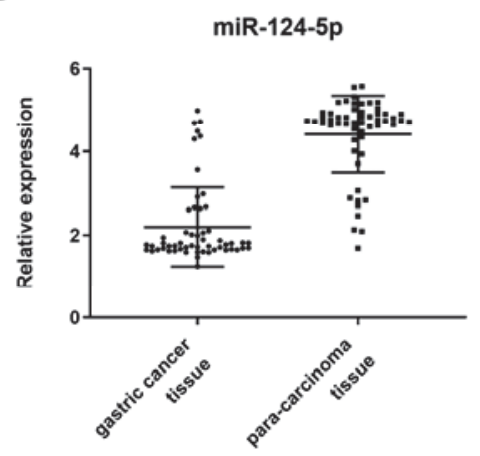

D

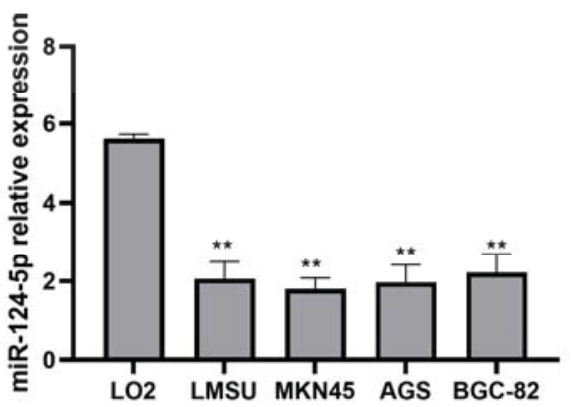

Figure 1: Expression of KCNQ1OT1 and miR-124-3p in gastric cancer A-B,RT-qPCR assessed relative expression of KCNQ1OT1 and miR-124-3p in both normal tissues $(n=55)$ and gastric tissues $(n=55)$. C-D, RT-qPCR was applied in cell lines (normal cell line LO2, gastric cancer cell lines AGS, BGC, Hep3B, LMSU and MKN45) for the relative expression of the genes KCNQ1OT1 and miR-124-3p. All the assays were done three times independently. ${ }^{*} \mathrm{P}<0.05$ 
A

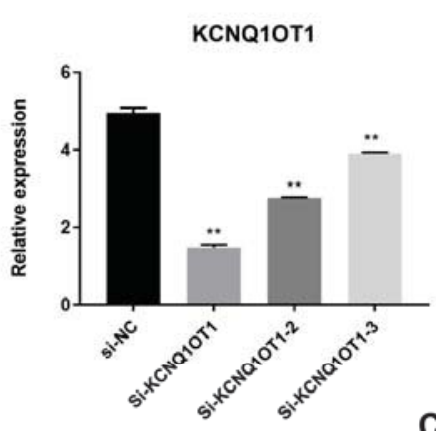

B

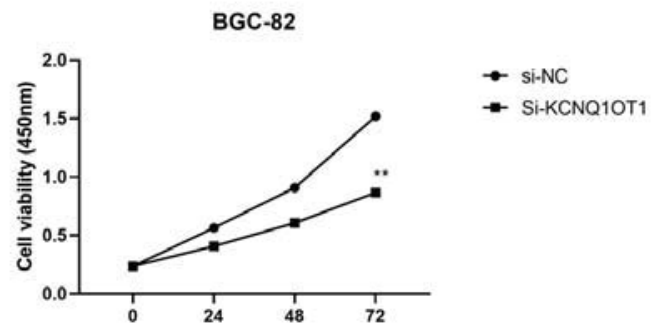

C

AGS

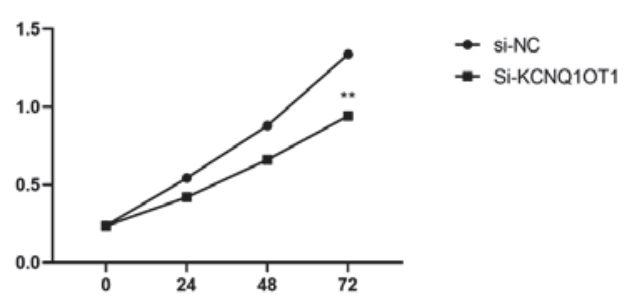

Figure 2: Downregulation of KCNQ1OT1 inhibited cell proliferation and cell viability in GC cells

A. siRNA1\#, siRNA2\# or siRNA3\# was transfected into the BGC-82 cell line. RT-qPCR was adopted to check the silencing efficiency of three different interference groups so as to select the most effective siRNA for the rest assays. Si-NC was a control group. B-C.CCK8 checked the cell viability of cells in si-NC group and si-KCNQ 1OT1 group in order to examine the effect of KCNQ1OT1 expression on cell viability in gastric cancer cell lines.All the assays were done for three independent times. ${ }^{* *} \mathrm{P}<0.05$

\section{Knockdown of KCNQ10T1 inhibited invasion of GC cells in vitro}

After we found that KCNQ1OT1 inhibited cell proliferation in gastric cancer cells, we wanted to study its other pro-malignant features. We performed transwell invasion assay to examine whether the KCNQ10T1 expression change would make a difference on the cellular invasive capabilities in GC. The results showed that KCNQ1OT1 siRNA1 transfected AGS and BGC cellsdecreased invasive capability when compared with the si-NC group (Figure $3 \mathrm{~A}$ and $3 \mathrm{~B})$. After this, we used western blot assays to detect the expression levels of EMT related genes which included $\mathrm{E}$-cadherin, $\mathrm{N}$-cadherin and Vimentin in AGS and BGC cells with knockdown of KCNQ10T1. As compared to the si-NC groups, E-cadherin was upregulated while $\mathrm{N}$-cadherin and Vimentin were reduced,which signified the inhibition of epithelial-mesenchymal transition (EMT) process (Figure 3B). These results showed that KCNQ1OT1 played a significant role in regulating cell invasion in GC.

\section{KCNQ10T1was a target of miR-124-3p}

miR- 124-3p was upregulated in AGS and BGC cells and RT-qPCR was applied to confirm miR124-3p expression level after transfection ,which was significantly upregulated in cells transfected with miR- 124-3p mimics rather than mimics-NC group (Figure 4A).

Bioinformatics tools Starbase (http://starbase. sysu.edu.cn) predicted the binding between miR124-3p and KCNQ1OT1 (Figure 4B). To further confirm whether KCNQ1OT1 was a target of miR-124-3p we performed a dual-luciferase assay and our results showed that the luciferase activity was greatly reduced in the wild-type and mimics group (Figure 4B). Therefore, it was sure of the binding between miR-124-3p and KCNQ1OT1. The RT-qPCR assays showed that knockdown of KCNQ1OT1 led to an increase in the expression of miR-124-2p in the cancer cells (Figure 4C). Our results therefore supported that KCNQ10T1 was a target of miR-124-3p and might regulatethe miR-124-3pexpression reversely.

\section{Downregulation of miR-124-3p reversed the effect of knockdown of KCNQ1OT1 on the gastric cancer cell lines}

miR-124-3p inhibitor was introduced to help observe the regulatory mechanism beneath KCNQ1OT1 in gastric cancer. As Figure 5A-B showed, knockdown of miR-124-3p could be reversed by si-KCNQ1OT1 in AGS and BGC-82 cell lines. Figure $5 C-D$ displayed that cell viability was inhibited by down-regulation of KCNQ1OT1 
A

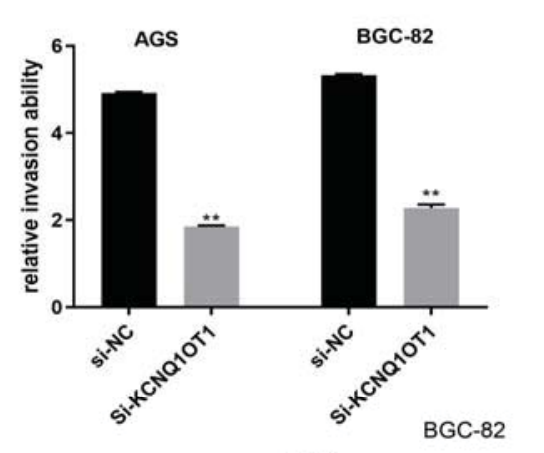

B

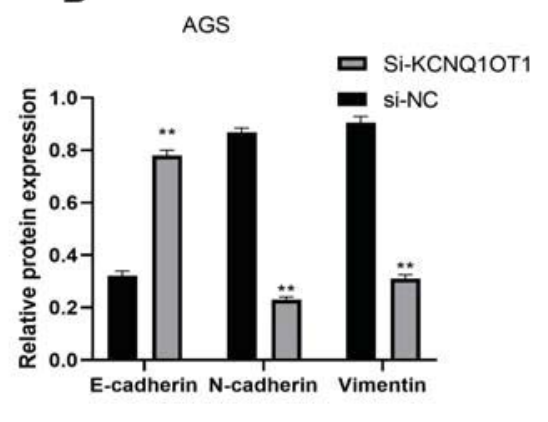

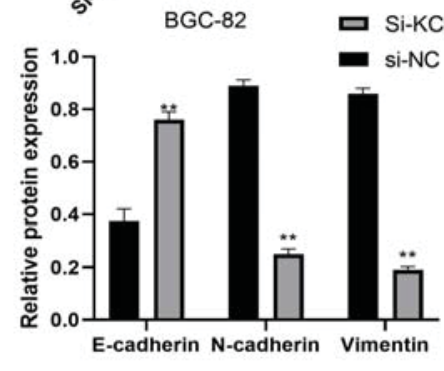

C

Figure 3: Knockdown of KCNQ1OT1 inhibited invasion of GC cells in vitro

AGS and BGC-82 cells transfected with si-NC or si-KCNQ1OT1 were selected for Transwell Invasion assays to study the cellular function of KCNQ1OT1 in gastric cancer. C. Western Blot assays were used to measure the relative protein expression of the key proteins involved in EMT process. E-cadherin, $\mathrm{N}$-cadherin and vimentin were checked in both cell lines. All the assays were conducted for three separate times. ${ }^{* *} \mathrm{P}<0.05$

A

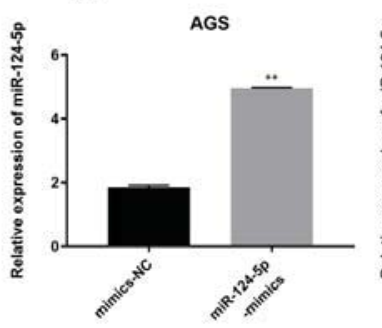

D

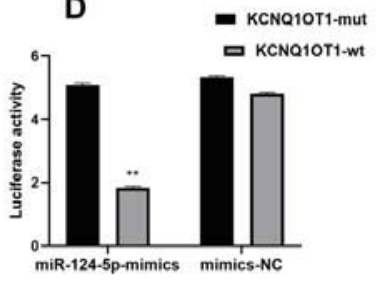

B

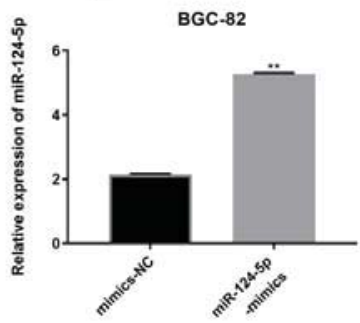

E

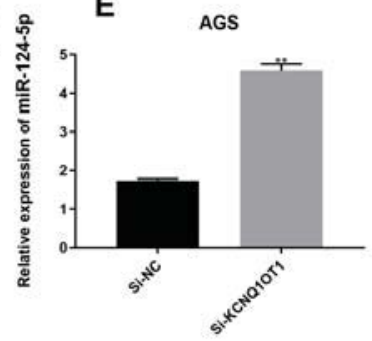

C

KCNQ1OT1: 5' ugggaggaugecUGCCUGCUGg 3'

| |||||| $\mid$

miR-124-3p : 3' ugacggacaga cACGGACGACa 5 '

Figure 4: KCNQ1OT1 was a target of miR-124-3p

B. miR-124-3p mimics and mimics-NC were transfected into AGS and BGC-82 cell lines and RT-qPCR checked the relative expression of miR-124-3p after transfection. C-D. Bioinformatics predicted the binding site between miR-124-3p and KCNQ1OT1 and Luciferase assay was performed to measure the luciferase activity after the AGS cells were co-transfected with KCNQ1OT1-mut, KCNQ1OT1-wt and miR-124-3p mimics or mimics NC. E. RT-qPCR checked the miR-124-3p expression after knocking down the KCNQ10T1 expression in both AGS and BGC-82 cell lines. All the assays were performed three times. ${ }^{* *} \mathrm{P}<0.05$ 

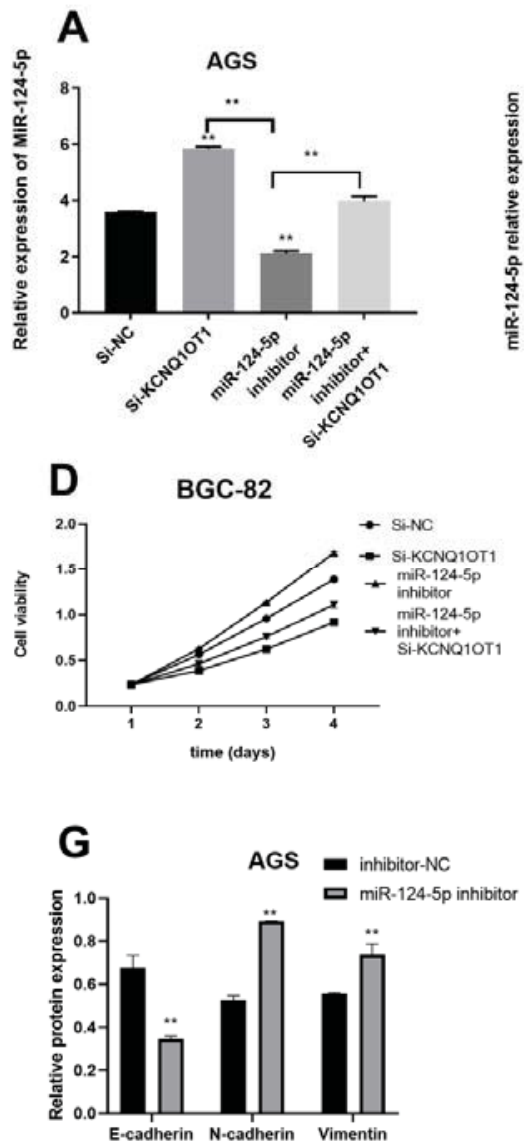
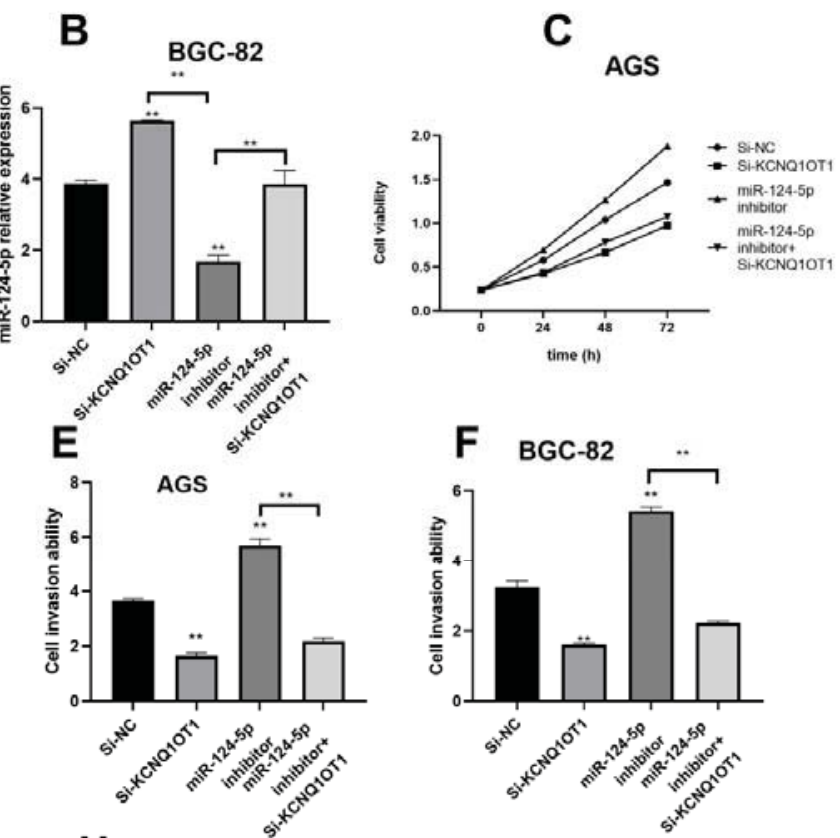

Figure 5: Downregulation of miR-124-3p reversed the effect of knockdown of KCNQ1OT1 on the gastric cancer cell lines

Four groups of cells in both AGS and BGC-82 cell lines were prepared for the following assays listed below: cells transfected with si-NC, si-KCNQ1OT1 or miR-124-3p inhibitor and cells co-transfected with si-KCNQ1OT1 and miR-124-3p inhibitor. A-B. RT-qPCR was conducted to check miR-124-3p expression in both cell lines after transfection. C-D.CCK8 assays were performed to show the cell viability.E-F.Transwell invasion assays were done to detect the cell invasion ability. G-H. Western Blot was for EMT biomarkers, E-cadherin, N-cadherin and vimentin. All the assays were performed three times. ${ }^{* *} \mathrm{P}<0.05$

and meanwhile it was enhanced by inhibition of miR-124-3p expression in the cancer cell lines. Moreover, the decrease by suppressed KCNQ10T1could be reversed by miR-124-3p inhibitor in the cell lines, which suggested that the miR-124-3p might have the opposite function on cell viability in gastric cancer. Furthermore, Transwell invasion was conducted so as to see whether there were similar changes as cell viability. Results showed that suppression of KCNQ10T1 could hinder cell invasion ability while inhibition of miR-124-3p could promote cell invasion(Figure 5E-F). In addition, KCNQ1OT1 effect could be inversed by miR-124-3p, which agreed with cell viability changes. Finally, EMT biomarkers were evaluated via Western Blot and the results were in accordance with cell viabilites and invasion discussed above.To be specific, the E-cadherin was inhibited by miR-124-3p inhibitor and promoted by si-KCNQ10T1 while N- cadherin and Vimentin had the opposite trends as E-cadherin, which suggested that downregulation of miR-124-3p could promote the EMT process and wheras decrease of KCNQ1OT1 expression could inhibit the EMT process. On the other hand, the results also pointed out that KCNQ10T1 could adjust the EMT through regulation of miR-124-3p.

\section{DISCUSSION}

Studies carried out recently have demonstrated that IncRNAs play different functions in various human diseases [11-13]. KCNQ1OT1 has been suspected to have a role in inhibiting tumor growth in diseases such as colorectal cancer [14] and lung cancer [15]. KCNQ1OT1 has also been reported to regulate proliferation and cisplatin resistance in tongue cancer [16]. Meanwhile, KCNQ1OT1 also controlled the expression of the 
imprinted genes that are found within the dominion of KCNQ1 [17]. Imprinted genes positively controlled apoptosis and restrain tumor growth [18, 19].However, there was no research that focused on the impact of KCNQ10T1 on gastric cancer progression yet.

Thus, our present study was dedicated to the exploration of how KCNQ10T1 would influence the gastric cancer and what the possible mechanism was. First, we traced 55 patients five years ago. Through qRT-PCR, it was observed that KCNQ10T1 was up-regulated in GC cells and tissues compared to normal ones, which suggested that KCNQ10T1 might be an oncogene in gastric cancer, which could possibly increase the growth and invasion of the cancer cells. To confirm the suggestion, we did CCK-8 and Transwell invasion assays and the results pointed out that silencing KCNQ10T1 could lead to decrease of cell viability and invasion ability. The results from our present study showed that KCNQ10T1 might promote cell proliferation and invasion in GC. The similar function has been reported by Ren where downregulation of KCNQ10T1 could suppress chemo-resistance induced by paclitaxel and to participate in inhibiting cell proliferation and invasion in lung adenocarcinoma cells [20]. Our results proposed that IncRNA KCNQ1OT1 acted as an oncogene.

EMT is a biological process where the malignant cells that have been derived from epithelium are transformed into mesenchymal cells and in the process invasion and migration abilities are enhanced [21, 22]. Epithelial markers of cells for example E-cadherin may be lost during EMT wheras the mesenchymal markers like vimentin, fibronectin, $\mathrm{N}$-cadherin are increased. What we found from our study suggested that KCNQ1OT1 played a significant role in regulating cell invasion in GC by downregulating the expression of the epithelial marker E-cadherin, and upregulating mesenchymal markers $\mathrm{N}$-cadherin and Vimentin, thus leading to high likelihood of the invasion of GC cells. On the other hand, several IncRNAs have demonstrated the ability to sponge miRNA thus promoting tumorigenesis. [23]. For instance, lincRNA-p21 negates the growth of non-small cell lung cancer through binding with miR-17-5p[24]. Further, Linc00152 was observed to confer oxaliplatin resistance in colon cancer via regulating miR-193a-3p [25].And the bioinformatics analysis showed the probable binding and hence luciferase was adopted to verify the idea. We found out that miR-124-3p was bound to KCNQ1OT1. MiR-124$3 p$ has been reported to suppress bladder cancer through targeting DNA methyltransferase 3B[26].
Furthermore, silencing KCNQ10T1 resulted in upregulating the expression of miR-124-3p, which indicated the correlation between KCNQ1OT1 and miR-124-3p and also supported the binding from a different angle. Therefore, it could be that upregulation of KCNQ1OT1 promoted cell viability, growth and invasion in gastric cancer cells by targeting and inhibiting miR-124-3p. It was previously demonstrated by Jin that KCNQ10T1 promoted cataractogenesis by competing with miR-214 and activating caspase-1 pathway [27]. Also, activation of KCNQ1OT1/miR-370/CCNE2 axis led to glioma carcinogenesis [28]. To further explore and validate the possible mechanism, we conducted CCK8,Transwell invasion and Western Blot for EMT biomakers to validate the independent effect of KCNQ10T1 or miR-124-3p on cell viability, invasion and EMT process. Discoveries supported our supposition, which proved that miR-124-3p could inhibit the cell viability, invasion and EMT process while KCNQ1OT1 could enhance the functions. Furthermore, when we co-transfected the si-KCNQ1OT1 and miR124-3p inhibitor into the cancer cell lines, the cellular functions were reversed compared to the singular transfection of si-KCNQ10T1, which indicated that KCNQ10T1 might exert its function on cells through regulation of miR-124$3 p$ expression.

\section{CONCLUSION}

It was concluded from the research that KCNQ10T1 could promote cell viability and invasion via targeting miR-124-3p,which indicated that KCNQ1OT1/miR-124-3p axis might be a potential therapeutic target for the treatment of gastric cancer..

\section{DECLARATIONS}

\section{Acknowledgement}

None provided

\section{Conflict of interest}

No conflict of interest is associated with this work.

\section{Contribution of authors}

We declare that this work was done by the authors named in this article and all liabilities pertaining to claims relating to the content of this article will be borne by the authors. 


\section{Open Access}

This is an Open Access article that uses a funding model which does not charge readers or their institutions for access and distributed under the terms of the Creative Commons Attribution License (http://creativecommons.org/licenses/by/ 4.0) and the Budapest Open Access Initiative (http://www.budapestopenaccessinitiative.org/rea d), which permit unrestricted use, distribution, and reproduction in any medium, provided the original work is properly credited.

\section{REFERENCES}

1. Wright NA, Poulsom R, Stamp G, Van Noorden S, Sarraf $C$, Elia G, Ahnen D, Jeffery R, Longcroft J, Pike C, et al.: Trefoil peptide gene expression in gastrointestinal epithelial cells in inflammatory bowel disease. Gastroenterology 1993, 104:12-20.

2. Torre LA, Bray F, Siegel RL, Ferlay J, Lortet-Tieulent J, Jemal A: Global cancer statistics, 2012. CA: a cancer journal for clinicians 2015, 65:87-108.

3. Xue $M$, Zhuo $Y$, Shan B: MicroRNAs, Long Noncoding RNAs, and Their Functions in Human Disease. Methods in molecular biology (Clifton, NJ) 2017, 1617:1-25.

4. Clark $M B$, Johnston $R L$, Inostroza-Ponta $M$, Fox $A H$, Fortini E, Moscato P, Dinger ME, Mattick JS: Genomewide analysis of long noncoding RNA stability. Genome research 2012, 22:885-898.

5. Rinn JL, Chang HY: Genome regulation by long noncoding RNAs. Annual review of biochemistry 2012, 81:145-166.

6. Arjumand W, Asiaf A, Ahmad ST: Noncoding RNAs in DNA Damage Response: Opportunities for Cancer Therapeutics. Methods in molecular biology (Clifton, NJ) 2018, 1699:3-21.

7. Cheng $Y$, Imanirad $P$, Jutooru I, Hedrick $E$, Jin $U H$, Rodrigues Hoffman A, Leal de Araujo J, Morpurgo B, Golovko A, Safe S: Role of metastasis-associated lung adenocarcinoma transcript-1 (MALAT-1) in pancreatic cancer. PloS one 2018, 13:e0192264.

8. Luo $X$, Qiu $Y$, Jiang $Y$, Chen $F$, Jiang $L$, Zhou $Y$, Dan $H$, Zeng $X$, Lei $Y L$, Chen Q: Long non-coding RNA implicated in the invasion and metastasis of head and neck cancer: possible function and mechanisms. Molecular cancer 2018, 17:14.

9. Yang $F$, Qin $Y, L v J$, Wang $Y$, Che $H$, Chen $X$, Jiang $Y, L i$ $A$, Sun $X$, Yue $E$, et al: Silencing long non-coding RNA Kcnq1ot1 alleviates pyroptosis and fibrosis in diabetic cardiomyopathy. Cell death \& disease 2018, 9:1000.

10. Pan YJ, Wei LL, Wu XJ, Huo FC, Mou J, Pei DS: MiR$106 a-5 p$ inhibits the cell migration and invasion of renal cell carcinoma through targeting PAK5. Cell death \& disease 2017, 8:e3155.

11. Wang $D$, Wang $D$, Wang $N$, Long Z, Ren X: Long NonCoding RNA BANCR Promotes Endometrial Cancer Cell Proliferation and Invasion by Regulating MMP2 and MMP1 via ERK/MAPK Signaling Pathway. Cellular physiology and biochemistry : international journal of experimental cellular physiology, biochemistry, and pharmacology 2016, 40:644-656.

12. Zhao L, Han T, Li Y, Sun J, Zhang S, LiU Y, Shan B, Zheng D, Shi J: The IncRNA SNHG5/miR-32 axis regulates gastric cancer cell proliferation and migration by targeting KLF4. FASEB journal : official publication of the Federation of American Societies for Experimental Biology 2017, 31:893-903.

13. He GY, Hu JL, Zhou L, Zhu XH, Xin SN, Zhang D, Lu GF,
Liao WT, Ding YQ, Liang L: The FOXD3/miR214/MED19 axis suppresses tumour growth and metastasis in human colorectal cancer. British journal of cancer 2016, 115:1367-1378.

14. Nakano S, Murakami $K$, Meguro $M$, Soejima $H$, Higashimoto K, Urano T, Kugoh H, Mukai T, Ikeguchi M, Oshimura M: Expression profile of LIT1/KCNQ1OT1 and epigenetic status at the KVDMR1 in colorectal cancers. Cancer science 2006, 97:1147-1154.

15. Soejima $H$, Nakagawachi $T$, Zhao W, Higashimoto $K$, Urano T, Matsukura S, Kitajima $Y$, Takeuchi $M$, Nakayama $M$, Oshimura $M$, et al: Silencing of imprinted CDKN1C gene expression is associated with loss of CpG and histone H3 lysine 9 methylation at DMR-LIT1 in esophageal cancer. Oncogene 2004, 23:4380-4388.

16. Zhang S, Ma $H$, Zhang $D$, Xie S, Wang W, Li Q, Lin Z, Wang $Y$ : LncRNA KCNQ1OT1 regulates proliferation and cisplatin resistance in tongue cancer via miR-211$5 p$ mediated Ezrin/Fak/Src signaling. Cell death \& disease 2018, 9:742.

17. Mohammad F, Mondal T, Guseva N, Pandey GK, Kanduri C: Kcnq1ot1 noncoding RNA mediates transcriptional gene silencing by interacting with Dnmt1. Development (Cambridge, England) 2010, 137:2493-2499.

18. Murakami K, Oshimura $M$, Kugoh H: Suggestive evidence for chromosomal localization of non-coding RNA from imprinted LIT1. Journal of human genetics 2007, 52:926-933.

19. Matsuoka S, Thompson JS, Edwards MC, Bartletta JM, Grundy P, Kalikin LM, Harper JW, Elledge SJ, Feinberg $A P$ : Imprinting of the gene encoding a human cyclindependent kinase inhibitor, p57KIP2, on chromosome 11p15. Proceedings of the National Academy of Sciences of the United States of America 1996, 93:3026-3030.

20. Ren $K, X u R$, Huang J, Zhao J, Shi W: Knockdown of long non-coding RNA KCNQ1OT1 depressed chemoresistance to paclitaxel in lung adenocarcinoma. Cancer chemotherapy and pharmacology 2017, 80:243-250.

21. Hay ED: An overview of epithelio-mesenchymal transformation. Acta anatomica 1995, 154:8-20.

22. Nieto MA: Epithelial plasticity: a common theme in embryonic and cancer cells. Science (New York, NY) 2013, 342:1234850.

23. Min A, Zhu C, Peng S, Rajthala S, Costea DE, Sapkota D: MicroRNAs as Important Players and Biomarkers in Oral Carcinogenesis. BioMed research international 2015, 2015:186904.

24. Ao $X$, Jiang $M$, Zhou J, Liang $H$, Xia $H$, Chen $G$ : lincRNAp21 inhibits the progression of nonsmall cell lung cancer via targeting miR175p. Oncology reports 2019, 41:789-800.

25. Yue B, Cai D, Liu C, Fang C, Yan D: Linc00152 Functions as a Competing Endogenous RNA to Confer Oxaliplatin Resistance and Holds Prognostic Values in Colon Cancer. Molecular therapy: the journal of the American Society of Gene Therapy 2016, 24:20642077.

26. Zo RB, Long Z: MiR-124-3p suppresses bladder cancer by targeting DNA methyltransferase $3 B$. Journal of cellular physiology 2018, 234:464-474.

27. Jin $X$, Jin $H$, Shi $Y$, Guo $Y$, Zhang $H$ : Long Non-Coding RNA KCNQ1OT1 Promotes Cataractogenesis via miR214 and Activation of the Caspase-1 Pathway. Cellular physiology and biochemistry : international journal of experimental cellular physiology, biochemistry, and pharmacology 2017, 42:295-305.

28. Gong W, Zheng J, Liu X, Liu Y, Guo J, Gao Y, Tao W, Chen J, Li Z, Ma J, Xue Y: Knockdown of Long NonCoding RNA KCNQ1OT1 Restrained Glioma Cells' Malignancy by Activating miR-370/CCNE2 Axis. Frontiers in cellular neuroscience 2017, 11:84 